\title{
XXVII. The transverse oscillations of a canal of circular section
}

\author{
Robert A. Houstoun M.A. Ph.D. D.Sc.
}

To cite this article: Robert A. Houstoun M.A. Ph.D. D.Sc. (1909) XXVII. The transverse oscillations of a canal of circular section, Philosophical Magazine Series 6, 17:98, 325-328, DOI: 10.1080/14786440208636605

To link to this article: http://dx.doi.org/10.1080/14786440208636605

曲 Published online: 21 Apr 2009.

Submit your article to this journal $₫$

Џll Article views: 2

Q View related articles $₫$ 
earth the action of the thorium family as well as that of the uranium family should be taken into consideration.

Strutt found that the average amount of radium present in the surface rocks was $5 \times 10^{-12}$ gram per c.c. Taking the life of thorium as $10^{9}$ years and that of radium as $2 \times 10^{3}$ years, we see from our previous values that there must be about $7 \times 10^{5}$ as much thorium as radium, $i$. $e$. there must be about $10^{-6}$ gram per c.c. This amount is of course still very small, only amounting to about $10^{-5}$ per cent. of the whole mass.

S. J. Allen, working in Pittsburg, Conn., has lately published a paper on "Radioactivity in a Smoke-laden Atmosphere"*. $\mathrm{He}_{\mathrm{e}}$ finds the same type of results for negatively charged wires and obtains no activity on positively charged ones. With wires, however, suspended in the atmosphere without charge, he obtains about 25 per cent. of the activity found on a negatively charged one.

Similar experiments were made in the present case during smoky fogs, and although the wires when drawn in were always covered by a thick layer of dirt, no activity was observed either on a positively charged wire, or on one which had been suspended in the atmosphere for six weeks without charge.

I wish to thank Prof. Rutherford for suggesting the experiments and for his valuable help and encouragement from time to time.

Physical Laboratory,

The University, Manchester.

Sept. 1908.

XXVII. The Transverse Oscillations of a Canal of Circular Section. By Roberm A. Houstoun, M.A., Ph.D., D.Sc. $\dagger$

1 HE problem of the transverse oscillations of water contained in a canal of circular section has not yet been solved, though Lord Rayleigh has made an approximate determination of the frequeney of the slowest mode by a general method for the case, where the surface is at the level of the axis $\ddagger$. The object of this note is to show how the same case may be solved by the substitution of a series.

Take the origin at the centre of the circle. Let the axis of $x$ be horizontal and the axis of $y$ be measured positively

* Phys. Rer. Dec. 1907.

$\dagger$ Communicated by Prof. A. Gray, F.R.S.

$\mp$ Lamb's Hydrodynamics, 3rd edition, \$256.

Phil. Mag. S. 6. Vol, 17. No. 98, Feb. 1909. 
upwards. Let $a$ be the radius of the section. Then $\phi$, the velocity potential, must satisfy the equation

$$
\frac{\partial^{2} \phi}{\partial c^{2}}+\frac{\partial^{2} \phi}{\partial y^{2}}=0 \text {. . . . . . . . }
$$

at all points within the semicircle, the condition

$$
\frac{\partial \phi}{\partial n}=0 \quad \text {. . . . . . . . }
$$

at all points on the semicircle and

$$
\frac{\partial^{2} \phi}{\partial t^{2}}+g \frac{\partial \phi}{\partial y}=0
$$

at all points on the surface.

The problem cannot be solved by transforming conformally the case of triangular section solved by Kirchhoff *, because when the triangle is transformed into the semicircle, the surface condition no longer holds.

Suppose that the time-factor is $e^{i \sigma t}$. Then the surface condition becomes

$$
\sigma^{2} \phi=g \frac{\partial \phi}{\partial y}
$$

Let $\psi$ be the stream function. T'hen, omitting the time factor, we may assume

$$
\phi+i \psi=\mathrm{A}_{0}+\mathrm{A}_{1}(x+i y)+\mathrm{A}_{2}(x+i y)^{2} \ldots \ldots
$$

If we write the constants $\mathrm{A}_{n}$ in the form $\mathrm{P}_{n}+i \mathrm{Q}_{n}$, we obtain

$$
\begin{aligned}
& \phi=\mathrm{P}_{0}+\Sigma_{1}^{\infty} r^{n}\left(\mathrm{P}_{n} \cos n \theta-\mathrm{Q}_{n} \sin n \theta\right), \\
& \psi=\mathrm{Q}_{0}+\Sigma_{1}^{\infty} r^{n}\left(\mathrm{Q}_{n} \cos n \theta+\mathrm{P}_{n} \sin n \theta\right) .
\end{aligned}
$$

This satisfies (1).

Expressing the surface condition in polars we obtain

$$
\text { and } \quad \begin{aligned}
\sigma^{2} \phi & =\frac{g}{r} \frac{\partial \phi}{\partial \theta} \text { for } \theta=0 . \\
\sigma^{2} \phi & =-\frac{g}{r} \frac{\partial \phi}{\partial \theta} \text { for } \theta=\pi .
\end{aligned}
$$

Substituting the expression for $\phi$, we find that the surface condition is satisfied, provided that

$$
\begin{aligned}
n \mathrm{Q}_{n}= & -\sigma^{2} \mathrm{P}_{n-1} / g . \quad \cdot \quad \cdot \quad \cdot \quad \cdot \quad \cdot . \\
& * \text { Ibid. } \$ 255 .
\end{aligned}
$$


Using (4) we find that

$$
\psi=Q_{0}+\Sigma_{1}^{\infty} r^{n}\left(P_{n} \sin n \theta-\frac{\sigma^{2}}{g} \frac{P_{n-1}}{n} \cos n \theta\right) .
$$

In order to satisfy (2), $\psi$ must be constant for $r=a$ and $\pi<\theta<2 \pi$. We may, without loss of generality, assume that $\psi-\mathrm{Q}_{0}=1$. Then writing $a^{n} \mathrm{P}_{n}=\mathrm{S}_{n}$ and $\sigma^{2} a / g=x$, we have

$$
\begin{aligned}
1=\mathrm{S}_{1} \sin \theta & +\mathrm{S}_{2} \sin 2 \theta \ldots \ldots \\
& \quad-x\left(\mathrm{~S}_{0} \cos \theta+\frac{\mathrm{S}_{1}}{2} \cos 2 \theta+\frac{\mathrm{S}_{2}}{3} \cos 3 \theta \ldots \ldots\right) .
\end{aligned}
$$

If the constants $x, \mathrm{~S}_{0}, \mathrm{~S}_{1}, \mathrm{~S}_{2}$, \&c. be chosen so that this equation holds for all values of $\theta$ between $\pi$ and $2 \pi$, then $\phi$ satisfies all the prescribed conditions.

Since the series on the right of equation (5) must be symmetrical about $\mathrm{OY}, \mathrm{S}_{0}=\mathrm{S}_{2}=\mathrm{S}_{4} \ldots \ldots=0$. The calculation of the other constants is extremely laborious, and we shall retain only the first three. To solve for $x, S_{1}, S_{3}$, and $S_{3}$, we require four equations. Since the series is symmetrical about $O Y$, it is necessary to equate it to unity only for the range $\pi<\theta<\frac{3 \pi}{2}$. Hence, putting $\theta=\pi$, $\pi+\frac{\pi}{6}, \pi+\frac{\pi}{3}$, and $\frac{3 \pi}{2}$ in succession, we obtain from

$$
\begin{aligned}
& 1=-x\left(\frac{S_{1}}{2}+\frac{S_{3}}{4}+\frac{S_{5}}{6}\right), \\
& 1=-\frac{S_{1}}{2}-S_{3}-\frac{S_{5}}{2}-x\left(\frac{S_{1}}{4}-\frac{S_{3}}{8}-\frac{S_{5}}{6}\right), \\
& 1=-\frac{S_{1} \sqrt{ } 3}{2}+\frac{S_{5} \sqrt{ } 3}{2}-x\left(-\frac{S_{1}}{4}-\frac{S_{5}}{8}+\frac{S_{5}}{6}\right), \\
& 1=-S_{1}+S_{3}-S_{5}-x\left(-\frac{S_{1}}{2}+\frac{S_{3}}{4}-\frac{S_{5}}{6}\right) .
\end{aligned} .
$$

Eliminating $\mathrm{S}_{1}, \mathrm{~S}_{3}, \mathrm{~S}_{5}$,

$$
\left|\begin{array}{rrrr}
1, & \frac{x}{2}, & \frac{x}{4}, & \frac{x}{6} \\
1, & \frac{1}{2}+\frac{x}{4}, & 1-\frac{e}{8}, & \frac{1}{2}-\frac{x}{6} \\
1, & \frac{\sqrt{ } 3}{2}-\frac{x}{4}, & -\frac{x}{8}, & -\frac{\sqrt{ } 3}{2}+\frac{x}{\overline{0}} \\
1, & 1-\frac{x}{2}, & -1+\frac{x}{4}, & 1-\frac{x}{6}
\end{array}\right| .
$$


328 Transverse Oscillations of a Canal of Circular Section.

The roots of this equation should give the frequencies of different modes. It has one real root, $x=1 \cdot 345$, and two complex ones. From $x=1 \cdot 345$ we obtain $\sigma=\sqrt{g / a} \cdot 1 \cdot 160$. Lord Rayleigh finds for the gravest mode, $\sigma=\sqrt{ } \frac{\sqrt{g / a}}{} 1 \cdot 1644$.

If we substitute $x=1.345$ in equations (6) we find

$$
\begin{aligned}
& S_{1}=-1 \cdot 764, \\
& S_{3}=\quad \cdot 5895, \\
& S_{5}=-.0538 .
\end{aligned}
$$

Hence

$$
\begin{aligned}
\psi=\left(\frac{r}{a}\right) \mathrm{S}_{1} \sin \theta & +\left(\frac{r}{a}\right)^{3} \mathrm{~S}_{3} \sin 3 \theta+\left({ }_{a}\right)^{5} \mathrm{~S}_{5} \sin 5 \theta-x\left(\left(\frac{r}{a}\right)^{2} \frac{\mathrm{S}_{1}}{2} \cos 2 \theta\right. \\
& \left.+\left(\frac{r}{a}\right)^{4} \frac{\mathrm{S}_{3}}{4} \cos 4 \theta+\left(\frac{r}{a}\right)^{6} \frac{\mathrm{S}_{5}}{6} \cos 6 \theta\right) \\
= & -\left(\frac{r}{a}\right) 1 \cdot 764 \sin \theta+\left(\frac{r}{a}\right)^{2} 1 \cdot 187 \cos 2 \theta+\left(\frac{r}{a}\right)^{3} \cdot 5895 \sin 3 \theta \\
& -\left(\frac{r}{a}\right)^{4} \cdot 1982 \cos 4 \theta-\left(\frac{r}{a}\right)^{5} \cdot 0538 \sin 5 \theta+\left(\frac{r}{a}\right)^{6} \cdot 0121 \cos 6 \theta .
\end{aligned}
$$

The accompanying figure represents the stream-lines calculated by the above formula. Since they are symmetrical about OY only one quadrant is given. Hence we see that with three constants we obtain a satisfactory representation of the slowest mode.

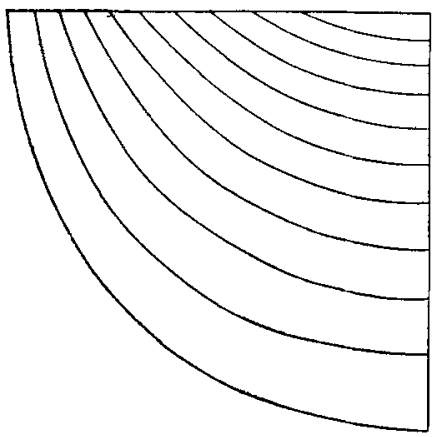

If we take more constants we obtain an equation of higher degree for $x$, and it seems that its other real roots may give the higher modes. I have, however, not been able to discover any way of discussing the general equation for $x$, and to calculate particular cases would be exceedingly laborious. 